2. Whitaker D, Henderson DW, Shilkin KB. The concept of mesothelioma in situ: implications for diagnosis and histogenesis. Semin Diagn Pathol 1992: 9: $151-61$

3. WHO Classification of Tumours Editorial Board. Thoracic Tumours. 5th ed. Lyon: International Agency for Reserach on Cancer, 2021.

4. Pulford E, Henderson DW, Klebe S. Malignant mesothelioma in situ: diagnostic and clinical considerations. Pathology 2020; 52: 635-42.

5. Minami K, Jimbo N, Tanaka Y, et al. Malignant mesothelioma in situ diagnosed by methylthioadenosine phosphorylase loss and homozygous deletion of CDKN2A: a case report. Virchows Arch 2020; 476: 469-73.

6. Churg A, Galateau-Salle F, Roden AC, et al. Malignant mesothelioma in situ: morphologic features and clinical outcome. Mod Pathol 2020; 33: 297-302.

7. Churg A, Dacic S, Galateau-Salle F, et al. Malignant mesothelioma in situ: clinical and pathologic implications. J Thorac Oncol 2020; 15: 899-901.

8. Klebe S, Nakatani Y, Dobra K, et al. The concept of mesothelioma in situ, with consideration of its potential impact on cytology diagnosis. Pathology 2021; 53: 446-53.

\section{Keratoameloblastomatous transformation of a recurrent unicystic ameloblastoma: a novel case raising diagnostic and classification difficulties}

Sir,

The origin and nature of keratoameloblastoma (KAM) and/or solid odontogenic keratocyst (SOKC) have never been studied comprehensively. In a recent issue of Pathology, Zhang et al. ${ }^{1}$ presented a series of KAM/SOKC cases and discussed their pathological profile and mutational status with an extensive review of the literature. The authors suggested that although the degree of transformation may vary, KAM should be classified as SOKC with ameloblastomatous changes. ${ }^{1}$ Their conclusion supported our own view that KAM and SOKC may share a histogenetic relationship. ${ }^{2,3}$ There is currently an overall agreement on the existence of the clinicopathological spectrum of KAM and SOKC. ${ }^{1}$ Here we present the first known reported case of unicystic AM (UAM) showing late recurrence as a mixture of follicular AM and KAM/SOKC. This rare curiosity invites two alternative interpretations: (1) UAM may be a novel histogenetic source of KAM/SOKC, and (2) KAM/SOKC ex UAM may represent part of the morphological spectrum of AMs.

A 32-year-old man was referred for treatment of a $2 \mathrm{~cm}$ radiolucent area in the premolar region of the left mandible. Radiographs revealed a well corticated, rounded unilocular cystic lesion extending from the incisor to the second premolar (Fig. 1A,B). It had caused thinning of the buccal cortex and resorbed the roots of the canine and premolars (Fig. 1B). The cyst aspirate contained dark liquid with no keratin debris (Fig. 1C). The cyst was treated by enucleation and curettage, and grossly was found to have a single cavity lined with a very thin wall of uniform thickness. Microscopically, it exhibited a simple lining of non-keratinised epithelium with focal areas of ameloblastic changes (a peripheral basal layer of columnar to cuboidal cells with hyperchromatic nuclei arranged in a palisading pattern with reverse polarity) and, despite a careful search, neither papillary projections into the lumen nor solid islands within the wall were evident, thus fulfilling the criteria for UAM of the luminal type (Fig. 1D-F). Approximately 5 years after surgery, radiographic examination yielded a multilocular lesion suggestive of recurrence (Fig. 2A). The tumour was managed by marginal resection. The surgical specimen lacked the UAM configuration and was diagnosed histologically as solid/multicystic AM of the follicular type, infiltrating deep into the bone (Fig 2B,C). The AM epithelium also exhibited the classic growth pattern of KAM/ SOKC, consisting of multiple keratinising cysts of various sizes with mural nests of acanthomatous AM (Fig. 2B,D,E). Notably, some of the lining epithelium showed ameloblastomatous transformation (Fig. 2F). At the invasive front, small follicles of KAM permeated widely between medullary bone trabeculae (Fig. 2G). Collectively, the recurrent lesion showed features of both acanthomatous change in a classic follicular AM with other areas of more classical $\mathrm{KAM} / \mathrm{SOKC}$. The patient has been followed for 7 years, and no sign of recurrence has been observed.

The term 'KAM' was coined by Pindborg ${ }^{4}$ in 1970 to describe a specific type of acanthomatous AM with microscopic features of keratinising cyst. Since then, many examples have appeared in the literature under a variety of names. ${ }^{1-3,5}$ Although there are well defined criteria for the histopathological diagnosis of KAM, ${ }^{5}$ this tumour was not recognised as a separate type of AM in the latest World Health Organization (WHO) classification, ${ }^{6}$ and the term 'KAM' itself has not been adopted. As evident in a review of SOKC by Zhang et al., many descriptive terms have been used, ${ }^{5,7}$ but in 2003 our group initially reported a recurrent $\mathrm{OKC}$ with invasive foci of KAM in the surrounding bone, applying the prefix 'solid-cystic tumour variant'. ${ }^{2}$ With the accumulation of published cases, in 2012 we also provided evidence that SOKC and KAM are likely related entities, representing a continuous spectrum of a single tumour type. ${ }^{3}$ In the OKC section of the 2017 WHO classification, there is only a brief comment on SOKC as an evolving entity, ${ }^{6}$ and unlike KAM, no formal publication has yet defined SOKC on the basis of reproducible diagnostic criteria. ${ }^{1}$ However, it is generally accepted that some OKCs are neoplastic in nature, and capable of transformation into AM, ameloblastic carcinoma or cystic squamous cell carcinoma reminiscent of carcinoma cuniculatum. ${ }^{8-10}$

In the present case, the primary lesion fitted the category of UAM clinically, radiologically and pathologically. ${ }^{6}$ This UAM recurred 5 years after conservative surgery and showed an unexpected shift in morphology. The recurrent tumour was of the follicular type characterised by the absence of UAM architecture and the presence of dominant and distinctive areas of KAM/SOKC. There has been only a single reported case of recurrent UAM, expressing an unusual histological transformation. ${ }^{11}$ The original UAM lesion recurred 23 years later in the form of granular/acanthomatous AM of the follicular type with invasive foci of squamous cell carcinoma, but with an absolute lack of KAM/SOKC features. There are two possible explanations for the natural history of the present lesion. The first is that, in addition to OKC, UAM may be another histogenetic source of KAM/SOKC. The second is that any change from UAM to KAM/SOKC may simply span a broad histological spectrum seen in AMs.

Considering that the histological subtypes of AM (including acanthomatous, granular and basaloid) are interchangeable in some circumstances and not permanent 

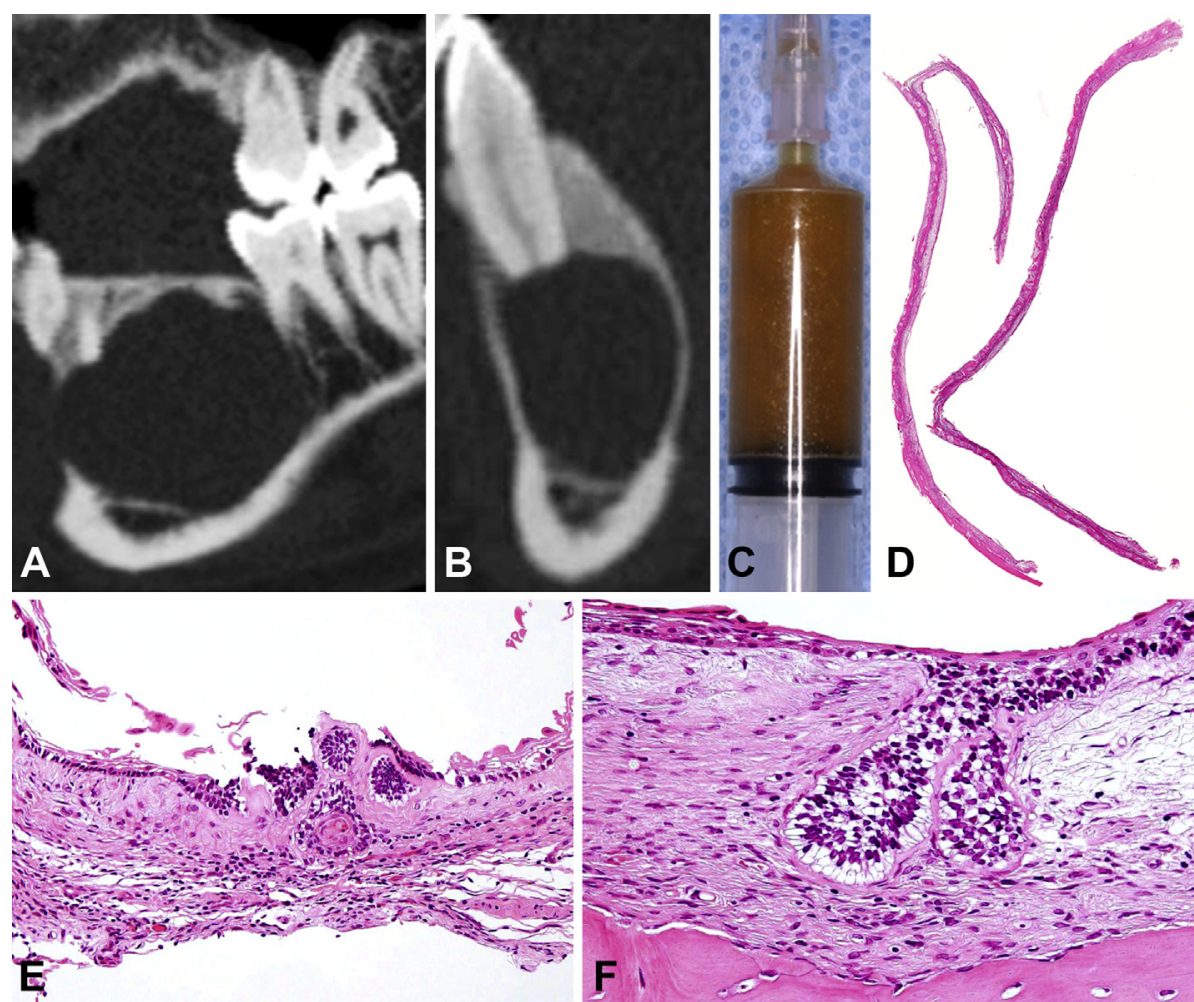

Fig. 1 (A,B) Computed tomography images of a well-defined unilocular radiolucency with root resorption. (C) Aspiration of significant amount of serosanguinous fluid. (D) Simple cyst with a very thin wall of uniform thickness. (E,F) Thin lining of non-keratinised epithelium with foci of ameloblastic changes and ameloblastomatous buddings.

features, ${ }^{6}$ we favour the second alternative. Indeed, the current case shows features of classic follicular AM with areas of acanthomatous changes merging into classical KAM/SOKC components.
In conclusion, we have described a case of UAM that showed follicular type recurrence and in which diagnostic morphological features of KAM/SOKC were evident. Our review of the literature did not reveal any previous report of

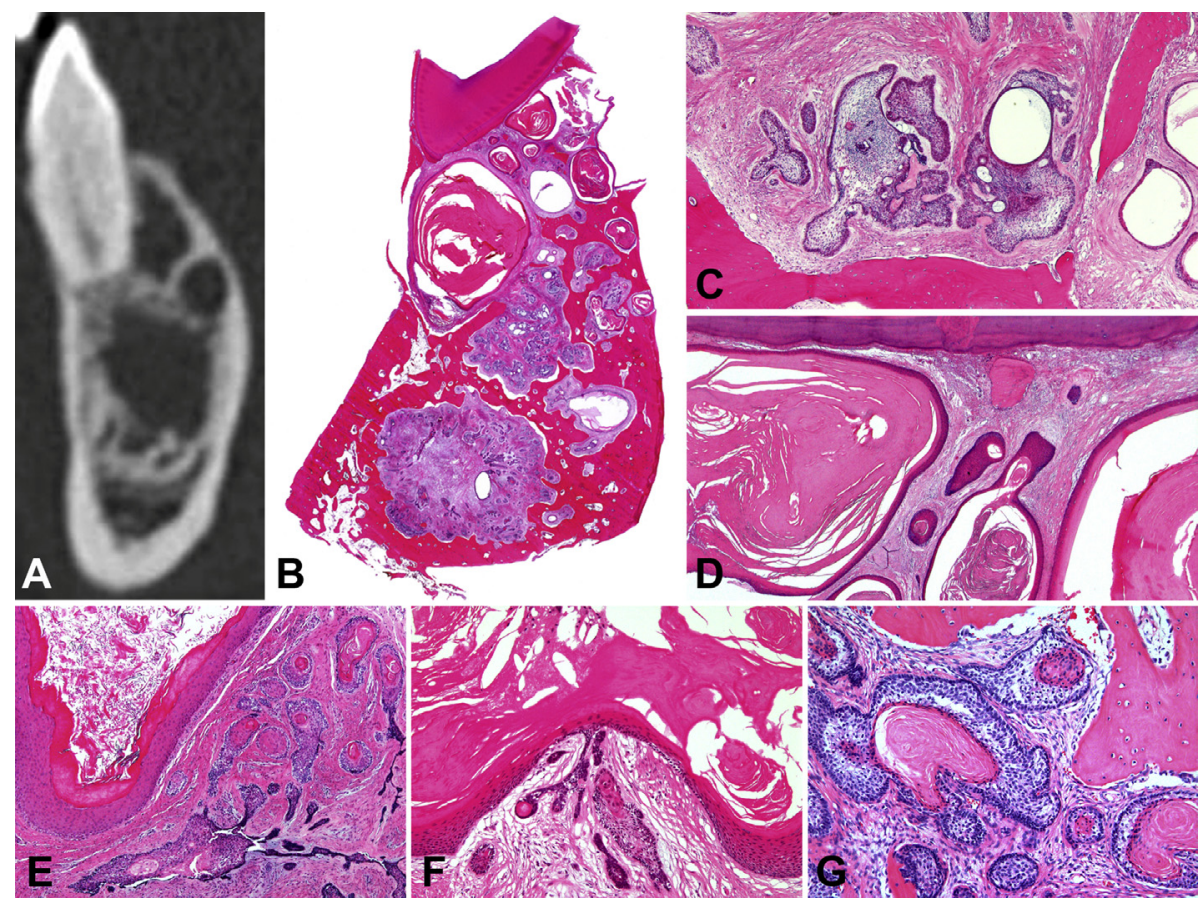

Fig. 2 (A) Computed tomography of a recurrent multilocular lesion. (B) The resected specimen showing a biphasic growth pattern of keratinising cyst and solid/ multicystic ameloblastoma. (C) Follicular ameloblastoma with microcyst formation in the central area of the tumour. (D) Large keratinising cysts extending close to the root. (E) Islands of acanthomatous ameloblastoma within the wall of a keratinising cyst. (F) Keratinising cyst with focal ameloblastomatous buddings. (G) Infiltrating small nests of keratoameloblastoma in the deeper part of the tumour. 
such a unique phenomenon in AMs. This case further supports the recent proposal that all types of AM constitute a continuous spectrum, necessitating revision of the basic concept that UAM is a distinct entity with defined clinicopathological characteristics. ${ }^{12}$ Additional studies should throw further light on this issue.

Conflicts of interest and sources of funding: The authors state that there are no conflicts of interest to disclose.

\section{Fumio Ide $^{1,2}$, Yumi Ito ${ }^{1}$, Michiko Nishimura ${ }^{2}$, Ikuko Ogawa ${ }^{3}$, Kentaro Kikuchi ${ }^{2}$}

${ }^{1}$ Department of Diagnostic Pathology, Tsurumi University Dental Hospital, Yokohama, Japan; ${ }^{2}$ Division of Oral Pathology, Department of Diagnostic and Therapeutic Sciences, Meikai University School of Dentistry, Saitama, Japan; ${ }^{3}$ Center of Oral Clinical Examination, Hiroshima University Hospital, Hiroshima, Japan

Contact Dr Yumi Ito.

E-mail: ito-yu@tsurumi-u.ac.jp

1. Zhang R, Yang J, Zhang J, Hong Y, Xie X, Li T. Should the solid variant of odontogenic keratocyst and keratoameloblastoma be classified as the same entity? A clinicopathological analysis of nine cases and a review of the literature. Pathology 2021; 53: 478-86.
2. Ide F, Mishima K, Saito I. Solid-cystic tumor variant of odontogenic keratocyst: an aggressive but benign lesion simulating keratoameloblastoma. Virchows Arch 2003; 442: 501-3.

3. Ide F, Ito Y, Muramatsu T, Saito I, Abiko Y. Histogenetic relations between keratoameloblastoma and solid variant of odontogenic keratocyst. Oral Surg Oral Med Oral Pathol Oral Radiol 2012; 114: 812-3.

4. Pindborg JJ, editor. Pathology of the Dental Hard Tissues. Copenhagen: Munksgaard, 1970; 371-6.

5. Whitt JC, Dunlap CL, Sheets JL, Thompson ML. Keratoameloblastoma: a tumor sui generis or a chimera? Oral Surg Oral Med Oral Pathol Oral Radiol Endod 2007; 104: 368-76.

6. El-Naggar AK, Chan JKC, Grandis JR, Takata T, Slootweg PJ, editors. WHO Classification of Head and Neck Tumours. 4th ed. Lyon: IARC, 2017; 235-6.

7. Janardhanan M, Rakesh S, Savithri V, Aravind T, Mohan M. Carcinoma cuniculatum of mandible masquerading as odontogenic keratocyst: challenges in the histopathological diagnosis. Head Neck Pathol 2021 15: $1313-21$

8. Slootweg PJ, Müller H. Malignant ameloblastoma or ameloblastic carcinoma. Oral Surg Oral Med Oral Pathol 1984: 57: 168-76.

9. Aldred M, Talacko A, Gordon A, Savage N, Shear M. Two cases of a previously undescribed odontogenic cyst/tumour variant. Pathology 2009; 41 (S1): 9-10.

10. Barrett AW, Garg M, Armstrong D, et al. Cystic squamous cell carcinomas of the jaws: twelve cases highlighting histopathological pitfalls. Int J Surg Pathol 2020; 28: 624-30.

11. Hijioka $\mathrm{H}$, Nishihara K, Suzuki $\mathrm{H}$, et al. Primary intraosseous squamous cell carcinoma arising from ameloblastoma of the mandible: a case report. J Oral Maxillofac Surg Med Pathol 2015; 27: 693-7.

12. Zlotogorski-Hurvitz A, Soluk Tekkesin M, Passador-Santos F, et al. Conceptual changes in ameloblastoma: suggested re-classification of a "veteran" tumor. Oral Dis 2021; Jan 6: https://doi.org/10.1111/odi.13770.

DOI: https://doi.org/10.1016/j.pathol.2021.11.007 